\title{
DINÁMICA DE LA EPIDEMIA DE MALARIA. PREDICCIONES DE SU TRAYECTORIA
}

\author{
Javier Rodríguez MD..$^{1^{*}}$ y Signed Prieto ${ }^{2}$ \\ ${ }^{1}$ Director del Grupo Insight Universidad Militar Nueva Granada, Centro de investigaciones \\ Clínica del Country. ${ }^{2}$ Investigadora Grupo Insight.
}

\begin{abstract}
Resumen
La teoría de los sistemas dinámicos estudia el estado y la evolución de los sistemas. La física newtoniana, en el contexto de las ecuaciones diferenciales de segundo orden, predice las trayectorias de los planetas, siendo este el primer sistema dinámico resuelto. La dinámica de las epidemias se ha asociado a diferentes factores causales como el clima, las migraciones y otros. Haciendo una analogía y utilizando el número de casos de malaria anual y la distancia (1960-2007), se hallaron la velocidad y la aceleración iniciales en rangos de tres años, y a partir de la ecuación diferencial de segundo orden para la aceleración, se hicieron predicciones de la trayectoria de la epidemia. Al graficarlas en coordenadas polares, se predijeron los rangos de valores en los que se encuentra la trayectoria de la epidemia. Se encontró que los valores de aceleración y velocidad iniciales estaban entre -6,66 y 9,22 y entre 1 y 21 respectivamente y se predijeron correctamente los rangos de valores de las trayectorias de la epidemia de malaria para el 2005, 2006 y 2007 a través de atractores circulares concéntricos. Este resultado muestra que esta es una metodología teórico-práctica universal de predicción de la trayectoria de la epidemia para cualquier año, sin necesidad de consideraciones causales. La ley diferencial acausal predice los rangos de la trayectoria de la dinámica, de forma útil para las decisiones de salud pública.
\end{abstract}

Palabras clave: malaria, epidemia, tendencias, salud pública

\section{DYNAMIC OF THE MALARIA EPIDEMIC. EPIDEMIC PATH PREDICTIONS}

\begin{abstract}
The theory of systems dynamics studies the state and evolution of systems. Newtonian physics in the context of differential equations of the second order, predicts the paths of the planets, which comprise the first dynamic system that has been solved. The epidemics dynamics has been associated to different causal factors such as climate, migration, and others. Making an analogy between proportions of the annual number of malaria cases and the distance, since 1960 to 2007, the initial velocity and acceleration of three years ranges were found; beginning with the second order differential equation for the acceleration, predictions of the epidemic path were made. These were plotted in polar coordinates, predicting the values ranges in which the epidemic path is located. It was found that the values of initial acceleration and initial velocity were between -6.66 to 9.22, and between 1 to 21 , respectively. This finding is a prediction of all possible trajectories of the epidemic for these years without causal considerations. The values' ranges of paths of the malaria epidemic in 2005, 2006 and 2007 were correctly predicted through concentric circular attractors. The differential acausal law predicts ranges of the dynamics path, useful for public health decisions.
\end{abstract}

Key words: malaria, epidemics, trends, public health

* Correspondencia: Javier Rodríguez grupoinsight2025@yahoo.es. Dirección postal: Tr. 3 № 49-00, Facultad de Medicina, Universidad Militar Nueva Granada. Teléfono: 45275 41, Bogotá, Colombia. Recibido: Octubre 29 de 2010 Aceptado: Diciembre 15 de 2010 
Rodríguez J., Prieto S.

\title{
DINÂMICA DA EPIDEMIA DE MALÁRIA. PREVISÕES DA SUA TRAJETÓRIA
}

\begin{abstract}
Resumo
A teoria de sistemas dinâmicos estuda o estado e a evolução dos sistemas. A Física newtoniana, no contexto das equações diferenciais de segunda ordem, prediz o caminho dos planetas, este é o primeiro sistema dinâmico resolvido. A dinâmica das epidemias tem sido associada a diferentes fatores causais tais como o clima, as migração e outros. Por analogia, utilizando o número de casos de malária anualmente e a distância (1960-2007), descobrimos a velocidade e a aceleração iniciais em intervalos de três anos, e partindo da equação diferencial de segunda ordem para a aceleração foram feitos previsões sobre a trajetória da epidemia. Quando plotados em coordenadas polares, foram estimados intervalos de valores onde encontra-se a trajetória da epidemia. Verificou-se que os valores de aceleração e velocidade inicial ficaram entre -6,66 e 9,22 e entre 1 e 21, respectivamente e previou-se corretamente o intervalo de valores da trajetória da epidemia de malária para 2005, 2006 e 2007 através de atratores circulares concêntricas. Este resultado mostra que esta é uma metodologia de predição teórica e prática universal da trajetória da epidemia em qualquer ano, sem considerações de causalidade. A lei diferencial acausal prevê o intervalo da trajetória da dinâmica, de forma útil para decisões de saúde pública.
\end{abstract}

Palabras-chave: malária, epidemiologia, tendências, saúde pública

\section{Introducción}

La malaria es una enfermedad parasitaria que aunque es conocida desde la antigüedad, se ha constituido en uno de los mayores problemas de salud pública a nivel mundial en la actualidad (1). La Organización Mundial de la Salud (OMS) estima que el número de muertes causadas por la malaria está alrededor de un millón de personas por año y ha aumentado en los últimos años (2). Actualmente en Colombia, 25 millones de personas están en riesgo de ser infectadas, principalmente por Plasmodium falciparum y Plasmodium vivax. Diferentes estudios han abordado las distintas causas que originan la dinámica de la malaria asociándola, entre otros, a factores como la dinámica del fenómeno de ENSO (El Niño Southern Oscilation) (3-6), la temperatura y la humedad $(7,8)$, el impacto de los predadores de insectos en los ecosistemas $(9,10)$, la calidad y la distribución del agua superficial y los sitios de reproducción de insectos (7). Por la multiplicidad de causa-efecto particulares de estas asociaciones, es difícil establecer las predicciones generales de la dinámica de la epidemia de malaria, que sean aplicables en el momento de tomar las decisiones en salud pública.

Por esto es necesario desarrollar metodologías de predicción objetivas, basadas en leyes matemáticas y físicas, que permitan comprender los fenómenos de forma acausal y general. Este tipo de metodologías ya han sido desarrolladas previamente para la evaluación del monitoreo fetal, donde se desarrolló un método diagnóstico a partir de la ley Zipf-Mandelbrot, que evita las variaciones en la lectura hecha por diferentes observadores que ocasionan dificultades en el diagnóstico $(11,12)$. También se ha utilizado en la evaluación del holter (13), en la caracterización de fenómenos inmunológicos (14-16) y diferentes estructuras anatómicas (1723). Basados en la caminata al azar probabilista aplicada al análisis de la dinámica geométrica del número de casos anuales de infectados, se han logrado hacer predicciones temporales de la epidemia de dengue (24) y de malaria en Colombia, y de HIV en Estados Unidos para el año 2007 (observaciones no publicadas), corroboradas por los datos del Instituto Nacional de Salud (INS) de Colombia con una exactitud de 90,4\%, $95,60 \%$, y $99,7 \%$ respectivamente. Del mismo modo, se desarrollaron predicciones espacio temporales para la epidemia de malaria, realizadas a partir de la ley de la entropía en municipios de Colombia para el año 2007, con una exactitud del 99,86\% (25). También se construyó una predicción de la dinámica de malaria con un $100 \%$ de exactitud, a partir del estudio probabilista de rangos de infectados por año (26).

La cinemática es la rama de la mecánica que describe el movimiento sin importar las causas que lo producen. 
Las leyes de la cinemática se desarrollaron "cuando el reino de la ley era incierto en la naturaleza" (27), los fenómenos físicos terrestres y celestes estaban separados, y las leyes que los describían eran inconexas entre sí; por un lado estaba Galileo, que formularía los fundamentos de la cinemática terrestre, y por otro Kepler hacía lo propio con los fenómenos celestes. El trabajo de Kepler consistía en encontrar una trayectoria cerrada alrededor del sol para solucionar el problema del movimiento retrógrado de Marte visto desde la Tierra (27). Buscó incansablemente que esta trayectoria fuera circular con el sol en el centro, como lo consideraba Copérnico en su concepción heliocéntrica; sin embargo, la trayectoria que halló era elíptica con el sol en uno de los focos, y al generalizar este hallazgo con los demás planetas se convirtió en lo que hoy se conoce como la primera ley del movimiento planetario: los planetas describen elipses alrededor del sol con éste en uno de sus focos. Su decepción ante este descubrimiento fue tal que lo expresó diciendo: "me he quedado con un montón de estiércol", por no encontrar la figura circular platónica perfecta que esperaba según su concepción. También descubrió la segunda y tercera ley de Kepler: el radio vector desde el sol al planeta barre áreas iguales en tiempos iguales y el cuadrado del período de revolución de un planeta es proporcional al cubo de la magnitud del eje mayor de la elipse (28).

Galileo, partiendo de la creación de un experimento ideal, en el que consideró a una esfera que recibía un impulso inicial para iniciar su movimiento sobre una superficie, e imaginando que tanto la esfera como la superficie estarían tan bien pulidas que la fricción entre las dos fuera nula, llegó a la conclusión de que la esfera se movería en línea recta con velocidad constante indefinidamente a menos que se le imprimiera un impulso que cambiara su movimiento, formulando así lo que hoy se conoce como la ley de inercia (29). También descubrió la ley del movimiento uniforme, -es decir con velocidad constante, y que es base fundamental de la relatividad especial- (30), el movimiento uniformemente acelerado, -cuya aceleración es constante-, $y$ la ley de caída libre de cuerpos, que dice que al dejar libre un cuerpo en el campo gravitatorio de la Tierra, su velocidad vertical aumenta proporcionalmente con el tiempo (31).

Las leyes cinemáticas celestes eran inconexas entre sí, como anotó Einstein: "estas normas no aportan una explicación causal. Son tres reglas lógicamente independientes que no revelan ninguna conexión interna entre sí. La tercera ley no puede ser simplemente transferida, en términos cuantitativos, a otros cuerpos centrales que no sean el sol (por ejemplo, no existe relación entre el periodo de revolución de un planeta en torno al sol y el de la luna alrededor de su planeta)" (32).

De esta forma, antes de Newton apenas existían resultados auténticos, que sustentaran la confianza en la existencia de una causalidad física ininterrumpida, pues como lo expresaría Einstein: "Sin duda, los grandes materialistas de la antigua Grecia habían insistido en que todos los sucesos materiales pueden explicarse por el movimiento estrictamente regulado de los átomos, sin admitir como causa independiente la voluntad de ninguna criatura viva. Y sin duda, a su modo, Descartes había recogido una vez más esa tradición. Pero sólo se trataba de una ambición audaz, del ideal problemático de una escuela de filósofos" (32). Así pues el objetivo de Newton era contestar a la pregunta "¿Existe alguna regla simple por la que sea posible calcular por completo los movimientos de los cuerpos celestes en nuestro sistema planetario, si se conoce, en un determinado momento, su estado dinámico?"(32). Finalmente resuelve Newton la pregunta dentro de un esquema teórico completo como el que desarrolló con sus tres leyes: la ley de la inercia, la ley de acción y reacción, y la segunda ley, que es un ejemplo explícito de ley diferencial: $F=\frac{d P}{d t}$, la fuerza ejercida sobre un cuerpo es igual al cambio del momentum del cuerpo respecto al tiempo, conocida familiarmente como: la fuerza $(\mathrm{F})$ es igual a la masa $(\mathrm{m})$ por la aceleración (a) (33). Al solucionar ecuaciones diferenciales como ésta, no sólo se obtienen las predicciones sobre la dinámica de las mareas (34) y de los cometas (35), la precesión de los equinoccios (36), y las diferentes trayectorias de los cuerpos, sino que además de este sistema teórico completo se deducen las leyes de Kepler (37-39) y de Galileo.

La concepción causal dominó la física hasta el desarrollo de la mecánica estadística. En esta teoría, en la que se estudian sistemas de un número de cuerpos del orden de $10^{23}$, no era posible determinar causalmente su dinámica, pues la teoría de gravitación universal definía la interacción de sólo dos puntos de masa, por ejemplo Sol-Tierra (37-39). Por esto se crearon conceptos como la temperatura, que es el promedio de la energía cinética de las moléculas. Así en esta teoría se empiezan a manejar conceptos relacionados con 
promedios y leyes de la naturaleza acausales como la entropía $(40,41)$. Posteriormente la mecánica cuántica (42) basada en la teoría de la probabilidad y en su principio de indeterminación, desarrolló una teoría acausal de la naturaleza contrapuesta a la creencia muy extendida de la determinación causal de los fenómenos, que proviene de la costumbre como lo muestra Schrödinger (43). Finalmente la teoría de los sistemas dinámicos desde el caos (44), los sistemas impredecibles $(45,46)$ y la universalidad de los sistemas $(47,48)$ también ha solucionado los problemas dinámicos desde la concepción acausal.

Esta teoría de los sistemas dinámicos $(49,50)$ básicamente estudia el estado y evolución de los sistemas; el estado generalmente es descrito de forma matemática por ecuaciones diferenciales, y su evolución, -hacia donde se dirige el sistema-, se representa por medio de atractores en espacios de fases. Existen tres tipos de atractores que representan la evolución de los sistemas, el punto, el ciclo y el caótico; los dos primeros predecibles y el último una figura irregular impredecible que ha sido estudiada por la geometría fractal; con esta geometría se han caracterizado formas de la naturaleza $(17-23,51,52)$ y con la teoría de sistemas dinámicos, diferentes tipos de dinámicas (13,44,53-56).

El propósito de este artículo es describir y predecir la trayectoria dinámica de la epidemia de malaria en Colombia durante los años 2005, 2006 y 2007 a partir de la ecuación diferencial de segundo orden para la aceleración respecto al tiempo.

\section{Metodología}

Los datos del número anual de infectados de malaria desde 1960 hasta 2007, se obtuvieron de la base de datos del Sistema de Vigilancia Epidemiológica (SIVIGILA) del INS de Colombia. Se halla el mínimo valor de estos datos y se calcula la proporción de todos los valores respecto al mínimo; con estas proporciones se hace una analogía con las distancias de la cinemática, posteriormente con esas distancias se hallan las velocidades iniciales para cada año con la fórmula clásica: $v_{0}=\frac{r}{t}$ donde $r$ es la distancia para un determinado año y $t$ es el tiempo, que es de un año para todos los cálculos. Luego se calculan las aceleraciones correspondientes a los intervalos de dos años consecutivos con los datos conocidos, estas aceleraciones se calculan con la fórmula clásica; $a=\frac{v_{f}-v_{i}}{t}$. Se toman rangos de tres años consecutivos de estas aceleraciones hallando en cada rango los valores de las aceleraciones máxima $\left(a_{\text {máx }}\right)$ y mínima $\left(a_{\text {min }}\right)$ para establecer las ecuaciones diferenciales que permitan hallar los rangos de predicción tanto de las velocidades como de distancias.

Así, para hallar los rangos de la velocidad se utiliza la ecuación diferencial $\frac{d v}{d t}=a$; al resolverla para el rango de valores entre $\mathrm{a}_{\text {mín }} \mathrm{y}_{\text {máx }}$, se obtienen las dos expresiones para las velocidades máxima y mínima $v_{\text {máx }}$ y $v_{\text {mín }}$ que permitirán hacer las predicciones de velocidad, igualmente se procede para la distancia $r$, cuya ecuación diferencial está dada por $\frac{d^{2} r}{d t^{2}}=a$.

Sabiendo que la solución de las ecuaciones diferenciales es:

Para los rangos de velocidad:

$$
\begin{aligned}
& v_{\min }=v_{0}+a_{\min } t \text { (Ecuación 1) } \\
& v_{\text {max }}=v_{0}+a_{\text {max }} t \text { (Ecuación 2) }
\end{aligned}
$$

Y para los rangos de distancias:

$$
\begin{aligned}
& r_{\min }=r_{0 \min }+v_{\min } t+\frac{1}{2} a_{\min } t^{2} \text { (Ecuación 3) } \\
& r_{\max }=r_{0 \max }+v_{\max } t+\frac{1}{2} a_{\text {max }} t^{2} \text { (Ecuación 4) }
\end{aligned}
$$

Se asumirá que las condiciones iniciales $\mathrm{r}_{0 \text { mín }}$ y $\mathrm{r}_{0_{\text {máx }}}$ son iguales a cero, pues como el fenómeno que hemos considerado es la dinámica de la epidemia en un año particular sin contar con los datos de infectados del año anterior, entonces se puede asumir que la condición inicial es cero sin que esto afecte la metodología propuesta.

Las expresiones encontradas para los rangos de la velocidad y de la distancia se utilizan para hacer la predicción de los años 2005, 2006 y 2007, estas predicciones se comparan posteriormente con los datos para estos años, ver anexo.

\section{Resultados}

Los valores de las distancias se encontraron entre 1 y 21 (tabla 1), así como las velocidades iniciales, pues el tiempo es de un año. Entonces al calcular $v_{0}=\frac{r}{t}$ obtenemos que tanto $v_{0}$ como $r$ tienen el mismo valor; 
TABLA 1. Las distancias son adimensionales por ser proporciones entre valores de números de infectados, las unidades de las velocidades son uno/año, y las de las aceleraciones son 1/año2.

\begin{tabular}{cccccccc}
\hline AÑO & DISTANCIAS & $\mathbf{V}_{\mathbf{0}}$ & $\mathbf{V}$ mín. & V máx. & ACELERACIÓN & $\mathbf{V}_{\mathbf{0}} \mathbf{A}$ mín. & A máx. \\
\hline 1960 & 1,0000 & 1,0000 & & & & & \\
1970 & 3,6667 & 3,6667 & 3,1111 & 4,8889 & $-0,5556$ & $-0,5556$ & 1,2222 \\
1980 & 6,3333 & 6,3333 & 4,6667 & 12,000 & $-0,5556$ & $-0,5556$ & 1,8889 \\
1985 & 6,2222 & 6,2222 & 0,5556 & 9,2222 & 0,1111 & $-5,6667$ & 3,0000 \\
1990 & 11,2222 & 11,2222 & 11,0000 & 29,6667 & $-0,1111$ & $-0,1111$ & 9,2222 \\
1995 & 20,7778 & 20,7778 & 3,1111 & 40,7778 & 6,6667 & $-5,8889$ & 6,6667 \\
2000 & 12,0000 & 12,0000 & 5,3333 & 12,8889 & $-6,6667$ & $-6,6667$ & 0,8889 \\
2002 & 16,6667 & 16,6667 & 10,6667 & 32,0000 & $-3,0000$ & $-3,0000$ & 7,6667 \\
2006 & 10,2834 & 10,2834 & 8,1719 & 11,5954 & $-2,1116$ & $-2,1116$ & 0,6558 \\
2007 & 10,9392 & 10,9392 & 8,8277 & 11,5952 & 0,6558 & $-2,1116$ & 0,6558 \\
\hline
\end{tabular}

Distancias, velocidad inicial (V0), rangos de velocidades (V mín. y V máx.) y aceleraciones (A mín. y A máx.).

Como ejemplo sólo se tomaron los datos de 10 años de la totalidad.

como el tiempo es la unidad, se puede obviar el tiempo en los valores de las magnitudes. La aceleración varía entre -6,6667 y 9,2222, las aceleraciones negativas son desaceleraciones de un año respecto al anterior así como en la cinemática.

La Figura 1 permite ver cómo el valor real se encuentra dentro del espacio entre los dos círculos concéntricos predichos, la predicción circular del 2007 está contenida dentro del 2006; todas las predicciones para los años evaluados se superpusieron (tabla 2 ).

Tomemos como ejemplo el cálculo del rango de distancias para el 2007, entonces, sabiendo que para este año las variables toman los valores:

$$
\begin{aligned}
& a_{\min }=-2,1116\left(1 / \mathrm{año}^{2}\right), a_{\max }=0,6558 \\
& \left(1 / \mathrm{año}^{2}\right), v_{\min }=8,8277(1 / \text { año }), \\
& v_{\max }=11,5952(1 / \text { año }) \\
& \left.r_{0 \min }=r_{\text {omax }}=0 \text { y } t=1 \text { (año }\right)
\end{aligned}
$$

Reemplazando en las ecuaciones 3 y 4 :

$$
\left.r_{\min }=\left(\frac{1}{2}\right)\left(-2,1116 !(1]^{2}\right)\right)+8,8277(1)+r_{0 \min }=7,77
$$

Así mismo con la ecuación 4, obteniéndose $r_{\max }=11,92$

De esta forma se limita el espacio en el que la trayectoria de la epidemia de malaria se desenvolverá, así, en particular para el 2007 se obtiene: $r_{\max }=11,92$ y $r_{\min }=7,772$, ver tabla 2 y figura $1 \mathrm{~b}$.

Para establecer las predicciones de los años 2005 y 2006 se procede equivalentemente.

Como los valores $r_{\text {máx. }} y r_{\text {mín }}$ son proporciones del número de infectados por año, tenemos que al hallar su equivalente en números de infectados se obtiene que por ejemplo para el año $2007, r_{\text {máx. }}$ que es igual a 11,92 representa 107.309 infectados y $r_{\text {mín. }}$ que es igual a 7,772 representa 69.947 infectados, y entre estos valores se encuentra el número de infectados conocido para 2007 que fue de 98.453 (datos no mostrados); igualmente se pueden obtener estos rangos de infectados para los demás años. Más que la exactitud de los resultados, se quería evidenciar la existencia de un orden acausal subyacente a la dinámica de una epidemia, que permite comprender de una forma más sencilla el fenómeno.

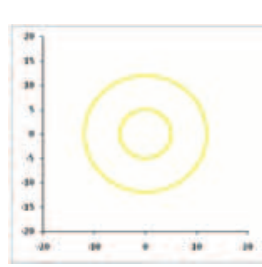

(a)

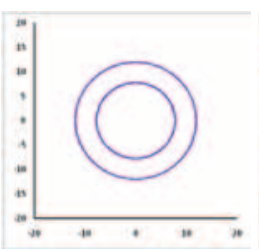

(b)

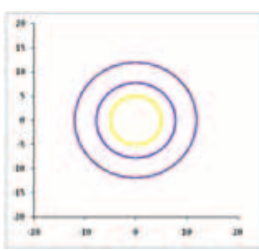

(c)
Figura 1. Predicción geométrica del espacio en el que se desenvolvería la trayectoria de la epidemia de malaria en Colombia. El círculo interior corresponde al $r$ mínimo, y el exterior al máximo: (a) para el 2006, (b) para el 2007, y (c) superposición del 2006 (amarillo) y 2007 (violeta), evidenciando la evolución de los rangos cinemáticos anuales de la trayectoria de la epidemia. Los valores de las abscisas y las ordenadas corresponden a distancias $(r)$ en analogía con el número de infectados por año. 
Tabla 2. Valores de r para las predicciones de los rangos: radio máximo $\left(r_{\text {maxx }}\right.$ PREDICHO) y mínimo $\left(r_{\text {min. }}\right.$ PREDICHO) y para los valores correspondientes al número de infectados de malaria registrados en la base de datos del SIVIGILA del INS de Colombia (rCONOCIDO)

\begin{tabular}{cccc}
\hline AÑO & $\begin{array}{c}\text { r máx. } \\
\text { PREDICHO }\end{array}$ & $\boldsymbol{r}$ CONOCIDO & $\begin{array}{c}\boldsymbol{r} \text { mín. } \\
\text { PREDICHO }\end{array}$ \\
\hline 2005 & 14,69 & 12,4 & 5,00 \\
2006 & 11,92 & 10,28 & 5,00 \\
2007 & 11,92 & 10,94 & 7,77 \\
\hline
\end{tabular}

Como se puede observar en la tabla 2 y en la figura 1 , se establece el espacio de posible ocupación de la dinámica de malaria para un año determinado, por lo tanto la predicción no es respecto a $r$ sino a dicho espacio. Al superponer estos espacios en el tiempo (figura 1c), se obtiene una representación que ofrece información útil para la toma de decisiones de salud pública.

\section{Discusión}

Este es el primer trabajo en el que se plantea la aplicación de la ley diferencial de la trayectoria de la epidemia de malaria en Colombia con ecuaciones diferenciales de segundo orden de la aceleración solucionada en rangos, encontrando círculos concéntricos con los $r_{\text {mín. }}$ y $r_{\text {máx. }}$ de las soluciones. Como en la mecánica newtoniana, en la teoría de gravitación universal, se tienen todas las proporciones de masas y distancias posibles en el contexto de una ley diferencial que determina el movimiento para todos los cuerpos densos del universo; así mismo en este trabajo también se predicen todas las posibles trayectorias de la epidemia que describen la dinámica encontrándose confinadas en círculos concéntricos, en analogía con las trayectorias de la cinemática y la dinámica planetaria, evidenciando un orden geométrico, físico, armónico y acausal subyacente a la dinámica de la epidemia, logrando una simplificación con una ley diferencial predictiva, útil en el momento de tomar decisiones de salud pública.

Varios estudios se han realizado con el objetivo de comprender la dinámica del número de infectados de malaria; sin embargo, al abordar este fenómeno desde la causalidad, se ha relacionado la epidemia de malaria con variados factores (3-10,56-59) como las poblaciones humanas, o del vector, los factores climáticos como la temperatura, regímenes de lluvias, fenómenos como ENSO, entre muchos. Esta multiplicidad causal presenta dificultades en el momento de querer establecer predicciones acerca de la dinámica, pues contrariamente al método de la física teórica, esta metodología no simplifica el fenómeno para establecer generalizaciones, sino que lo reduce a diferentes relaciones particulares. Predicciones acausales desde la física teórica ya se han realizado en malaria, con un porcentaje de exactitud para el año 2007 del 99,86\% (25) para municipios colombianos, y de un $100 \%$ con probabilidades de rangos de infectados para el mismo año (26), mientras que con la caminata al azar probabilista se lograron porcentajes de exactitud superiores al $90 \%$ para la epidemia de malaria y dengue en Colombia (24) y de HIV en Estados Unidos para el año 2007 (resultados no publicados).

Las ecuaciones de las trayectorias que se originan por la ley de gravitación universal corresponden a las cónicas, con las cuales se puede predecir el movimiento. En este trabajo, con todas las simplificaciones logradas y con la aplicación de conceptos $y$ leyes universales, se establecen ecuaciones diferenciales de segundo orden cuyas predicciones son varias trayectorias dentro de rangos acotados "de la inercia de la epidemia", generando una nueva comprensión geométrica acausal de las epidemias. A lo largo de la historia de la ciencia teórica, puede observarse el papel esencial de la geometría en la forma de contemplar la naturaleza, pues al cambiar la geometría, se han producido nuevas teorías; por ejemplo, con la geometría euclidiana se desarrolla la mecánica de Newton (60); la teoría de la relatividad de Einstein se va a expresar de manera óptima con la geometría riemniana (61), y la geometría fractal abrió paso para el estudio de las formas irregulares de la naturaleza (52) y de los sistemas dinámicos $(44,47,48,53,62,63)$. En esta investigación la geometría circular concéntrica crea una visión diferente de la realidad de la epidemia.

La forma en que se observa la dinámica de la epidemia de malaria en este trabajo es una visión platónica circular y estética, derivada de las ecuaciones obtenidas. Así, si observamos las ecuaciones 3 y 4 , vemos que todas las variables dinámicas involucradas en el lado derecho de las ecuaciones, tienen valores constantes para cada año (tabla 1), entonces la magnitud de la distancia será igual a una constante, es decir $r=c t e$. Esta ecuación corresponde a la ecuación de un círculo 
en coordenadas polares, así que teniendo los rangos, lo que obtenemos es: $r_{\max }=c t e_{\mathbf{1}}$ y $r_{\min }=c t e_{\mathbf{2}}$, que en el espacio cartesiano corresponde a dos círculos concéntricos (ver figura 1), de esta manera el espacio en el que se mueve la epidemia queda acotado, permitiendo así establecer predicciones de la trayectoria de la epidemia, estableciendo una nueva metodología física simple para predecir la evolución de una epidemia. Las predicciones han resultado sólidas en los rangos de años estudiados; sin embargo, es posible que las predicciones necesiten refinamientos de la teoría para ser aplicada a más de tres años, debido al posible comportamiento caótico determinista de la dinámica de la epidemia.

La física teórica actual, la mecánica cuántica (42), la teoría de sistemas dinámicos (47-50) y la mecánica estadística (41) predicen los fenómenos de la naturaleza desde la acausalidad; mientras que desde un punto de vista causal, el fenómeno de las epidemias se convierte en un fenómeno con dificultades predictivas por su multiplicidad de causas-efectos generando atractores caóticos. En este trabajo la simplicidad del estado y evolución del sistema se expresa en la ley diferencial y en las soluciones circulares concéntricas respectivamente. De este modo se obvian todas las dificultades ocasionadas por el análisis causal de la dinámica de la epidemia, que requieren información de cinco a siete años para la construcción de corredores epidemiológicos, y aún así presentan dificultades para su aplicación predictiva en todos los casos (64). En contraposición, mediante esta metodología se evidencia un orden matemático independiente de las causas, que simplifica la evaluación de la dinámica y permite establecer predicciones de utilidad en la toma de decisiones de salud pública independientemente de los factores de causalidad que sustentan las predicciones en este campo, tales como los relacionados con movimientos migratorios poblacionales, clima, régimen de lluvias, etc. (3-10,56-59). La predicción de la evolución de la epidemia reveló un atractor diferente, círculos concéntricos, a los conocidos: el punto, el ciclo y el caótico. Investigaciones en esta misma línea se han desarrollado en diferentes campos como el de la biología molecular y la inmunología (14-16), en morfología cardiaca $(17,18,20)$ y su dinámica, permitiendo además desarrollar metodologías de ayuda diagnóstica de la dinámica cardiaca en fetos y en adultos (11-13).

Entre la posición de Einstein de "Dios no juega a los dados" y la de Bohr "El universo es probabilista e indeter- minado" (65), proponemos una nueva concepción determinista - indeterminista de contemplar la naturaleza, teniendo relaciones de determinación-indeterminación simultáneamente, logrando predicciones acotadas del fenómeno como los círculos concéntricos encontrados.

De este trabajo se puede concluir que: 1- las ecuaciones diferenciales predicen los rangos, estableciendo los valores mínimos y máximos de la dinámica de la epidemia, como se observa en la geometría de los círculos concéntricos hallados, en los que es posible establecer predicciones útiles para las decisiones de salud pública; 2 - una nueva metodología deterministaindeterminista acausal de evaluación de la epidemia de malaria fue desarrollada, sin tener en cuenta sus causas y 3- los conceptos de aceleración y velocidad aplicados a los rangos constituyen una metodología que permite estudiar la mecánica de la epidemia.

\section{Agradecimientos}

A las doctoras Salua Osorio y Viviana Cerón y al INS de Colombia por facilitarnos los datos del SIVIGILA para realizar los cálculos y por apoyar la creatividad del grupo INSIGHT.

\section{Dedicatoria}

Al maestro Newton por dibujar la pintura de la armonía cósmica del universo. A los Hijos del Grupo Insight y a nuestro país que son por los que hacemos todo esto. A nuestros sobrinos.

\section{Referencias}

1. Sachs J, Malaney P. The Economic and Social Burden of Malaria. Nature. 2002;415:680-5.

2. World Health Organization. Guidelines for the Treatment of Malaria 2006.

3. Bouma M J, Van der Kaay H J. Epidemic Malaria in India and El Niño Southern Oscillation. Lancet. 1994;344:1638-9.

4. Bouma M J, Dye C. Cycles of Malaria Associated with El Niño in Venezuela. JAMA. 1997;278:1772-4.

5. Poveda G, Rojas W. Impacto del Fenómeno del Niño sobre la Intensificación de Malaria en Colombia. Memorias XII Seminario Nacional de Hidráulica e Hidrología; 1996. p. 647-654.

6. Poveda G, Rojas W. Evidences of the Association between Malaria Outbreaks in Colombia and the El Niño Southern Oscillation. Rev Acad Colom Cienc. 1997;21(81):421-9.

7. Patz J A, Epstein P R, Burke T A, Balbus J M. Global Climate Change and Emerging Infectious Diseases. JAMA. 1996;275:217-23.

8. Martens W, Jetten T H, Focks D A. Sensitivity of Malaria, Schistosomiasis and Dengue to Global Warming. Climatic Change. 1997;35:145-56.

9. Unninayar S S, Sprigg W. Climate and the Emergence and Spread of Infectious Diseases. EOS. 1995;76(47):478.

10. Epstein P R, Chikwenhere G P. Biodiversity Questions (Letter). Science. 1994;265:1510-11. 
11. Rodríguez J. Dynamical Systems Theory and Zipf - Mandelbrot Law Applied to the Development of a Fetal Monitoring Diagnostic Methodology. Proceedings of the XVIII FIGO World Congress Of Gynecology And Obstetrice. November 2006, Kuala Lumpur, Malaysia.

12. Rodríguez J, Prieto S, Ortiz L, Bautista A, Bernal P, Avilán N. Diagnóstico Matemático de la Monitoria Fetal Aplicando la Ley de Zipf-Mandelbrot. Rev Fac Med Univ Nac Colomb. 2006;54(2):96-107.

13. Rodríguez J, Prieto S, Avilán N, Correa C, Bernal P, Ortiz L, Ayala J. Nueva Metodología Física y matemática de Evaluación del Holter. Rev Colomb Cardiol. 2008;15:50-4.

14. Rodríguez J. Comportamiento Fractal del Repertorio T Específico Contra el Alergeno Poa P9. Rev Fac Med Univ Nac Colomb. 2005;53(2):72-8.

15. Rodríguez J. Teoría de Unión al HLA Clase II: Teoría de Probabilidad, Combinatoria y Entropía Aplicadas a Secuencias Peptídicas. Inmunología. 2008;27(4):151-66.

16. Rodríguez J. Caracterización Física y Matemática de Péptidos de Alta Unión de MSP-1 Mediante la Aplicación de la Teoría de la Probabilidad y la Entropía. Archivos de Alergia e Inmunología Clínica. 2008;39(2):74-82.

17. Rodríguez J, Mariño M, Avilán N, Echeverri D. Medidas Fractales de Arterias Coronarias en un Modelo Experimental de Reestenosis. Armonía Matemática Intrínseca de la Estructura Arterial. Rev Colomb Cardiol. 2002;10(2):65-72.

18. Rodríguez J, Álvarez L, Mariño M, Avilán G, Prieto S, Casadiego E, et al. Variabilidad de la Dimensión Fractal del Árbol Coronario Izquierdo en Pacientes con Enfermedad Arterial Oclusiva Severa. Dinámica Fractal de la Ramificación Coronaria. Rev Colomb Cardio. 2004;11(4):185-92.

19. Rodríguez J, Prieto S, Ortiz L, Avilán N, Álvarez L, Correa C, Prieto I. Comportamiento Fractal del Ventrículo Izquierdo Durante la Dinámica Cardíaca. Rev Colomb Cardiol. 2006;13(2):165-70.

20. Rodríguez J, Prieto S, Ortiz L, Correa C, Álvarez L, Bernal P, et al. Variabilidad de la Dimensión Fractal de la Ramificación Coronaria Izquierda en Ausencia y Presencia de Enfermedad Arterial Oclusiva Moderada y Severa. Rev Colomb Cardiol. 2007;14(3):173-80.

21. Rodríguez J, Correa C, Prieto S, Ospino B, Bernal P, Ortiz L, et al. Caracterización Geométrica de los Glóbulos Rojos. Diferenciación de Muestras Normales y Patológicas. Rev Cienc Salud. 2008;6(3):47-56.

22. Rodríguez J, Prieto S, Ortiz L, Wiesner C, Díaz M, Correa C. Descripción Matemática con Dimensiones Fractales de Células Normales y con Anormalidades Citológicas de Cuello Uterino. Rev Cien Salud. 2006;4(2):58-63.

23. Rodríguez J, Lemus J, Serrano J, Casadiego E, Correa C. Medidas Fractales Cardiotorácicas en Radiografía de Tórax. Rev Col Cardiol. 2005;12(3):129-34.

24. Rodríguez J, Correa C. Predicción Temporal de la Epidemia de Dengue en Colombia: Dinámica Probabilista de la Epidemia. Revista de Salud Pública. 2009;11(3):443-53.

25. Rodríguez J. Método para la Predicción de la Dinámica Temporal de la Malaria en los Municipios de Colombia. Rev Panam Salud Pública. 2010;27(3):211-8.

26. Rodríguez J. Dinámica Probabilista Temporal de la Epidemia de Malaria en Colombia. Revista Med. 2009;17(2):214-21.

27. Einstein A. Sobre la Teoría de la Relatividad y Otras Aportaciones Científicas. Madrid: Sarpe; 1983. p. 70-74.

28. Kleppner D, Kolenkow R. Central Force Motion: An Introduction to Mechanics. Singapore: Mc. Graw-Hill; 1973. p. 377-408.
29. Rañada A, García J, Yuste M. El Movimiento. En: Física Básica I. Madrid: Alianza Editorial S.A.; 1993. p. 44-89.

30. Yilmaz H. Galilean Relativity. En: Introduction to the Theory of Relativity and the Principles of Modern Physiscs. New York: Blaisdell Publishing Company; 1965. p. 1-8.

31. Einstein A. Notas Sobre el Origen de la Relatividad General. En: Einsteins A. Sobre la Teoría de la Relatividad y Otras Aportaciones Científicas. Madrid: Sarpe; 1983. p. 94-98.

32. Einstein A. La Mecánica de Newton y su Influencia en el Desarrollo de la Física Teórica. En: Einstein A. Sobre la Teoría de la Relatividad y Otras Aportaciones Científicas. Madrid: Sarpe; 1983. p. 61-69.

33. Rañada A, García J, Yuste M. Las Leyes de Newton. En: Rañada A. Física Básica I. Madrid: Alianza Editorial S.A.; 1993. p. 97-101.

34. Newton I. Proposiciones. En: Principios Matemáticos de la Filosofía Natural. Libro III. Barcelona: Altaya S.A.; 1993. p. 504-510.

35. Newton I. Lema I V. En: El Movimiento de los Nodos de la Luna. Libro III. Principios Matemáticos de la Filosofía Natural. Barcelona: Altaya S.A.; 1993. p. 561-616.

36. Newton I. Proposición XXXIX. Problema XX. En: El Movimiento de los Nodos de la Luna. Libro III. Principios Matemáticos de la Filosofía Natural. Barcelona: Altaya S.A.; 1993. p. 559-561.

37. Feynman R P, Leighton R B, Sands M. Capítulo 7: La teoría de la Gravitación. En: Física. Vol. 1. Segunda edición. Wilmington: Addison-Wesley Iberoamericana S. A.; 1987. p. 7.1-7.16.

38. Feynman R P, Leighton R B, Sands M. Capítulo 8: El Movimiento. En: Física. Vol. 1. Segunda edición. Wilmington: AddisonWesley Iberoamericana S. A.; 1987. p. 8.1-8.11.

39. Feynman R P, Leighton R B, Sands M. Capítulo 9: Leyes de la Dinámica de Newton. En: Física. Vol. 1. Segunda edición. Wilmington: Addison-Wesley Iberoamericana S. A.; 1987. p. 9.1-9.10

40. Matvéev A. Física molecular. Moscú: MIR; 1987.

41. Tolman R. Principles of statistical mechanics. New York: Dover Publications; 1979.

42. Feynman R P, Leighton RB, Sands M. Física. Vol. 3. Segunda edición. Wilmington: Addison-Wesley Iberoamericana, S. A.; 1987.

43. Schrödinger E. ¿Qué es una Ley Física? Rev Cultural de Occidente. 1968;11(4):375-84.

44. Lorenz E N. Deterministic Nonperiodic Flow. J Atmos Sci. 1963;20:130-41.

45. Grassberger P, Procaccia I. Characterization of Strange Attractors. Phys Rev Lett. 1983;50:346-9.

46. Ruelle D, Takens F. On the Nature of Turbulence. Commun Math Phys. 1971;20:167-92.

47. Feigenbaum M J. Quantitative Universality for a Class of Nonlinear Transformations. J Stat Phys. 1978;19(1):25-52.

48. Feigenbaum M J. Universal Behavior in Nonlinear Systems. Physica. 7D;1983.

49. Rasband S N. Chaotic Dynamics of Nonlinear Systems. John Wiley \& Sons, New York, 1990.

50. Guckenheimer J, Holmes P. Nonlinear Oscillations, Dynamical Systems and Bifurcations of Vector Fields. New York: SpringerVerlag; 1983.

51. Peitgen $H$, Jürgens $H$, Saupe D. Length, Area and Dimension: Measuring Complexity and Scaling Properties. En: Chaos and Fractals: New Frontiers of Science. New York: Springer-Verlag; 1992. p. 183-228.

52. Mandelbrot B. The Fractal Geometry of Nature. San Francisco: Freeman; 1972. 
53. Lorenz E N. The Local Structure of a Chaotic Attractor in Four Dimensions. Physica. 13D; 1984.

54. Peitgen $H$, Jürgens $H$, Saupe D. The backbone of fractals. En: Chaos and Fractals: New Frontiers of Science. N.Y.: SpringerVerlag; 1992. p. 15-62.

55. Huikuri H V, Mäkikallio T H, Peng Ch, Goldberger A L, Hintze U, Moller M. Fractal Correlation Properties of R-R Interval Dynamics and Mortality in Patients with Depressed Left Ventricular Function after an Acute Myocardial Infartion. Circulation. 2000;101:47-53

56. Briët O, Vounatsou P, Gunawardena D M, Galappaththy G, Amerasinghe P H. Models for Short Term Malaria Prediction in Sri Lanka. Malaria Journal. 2008;7:76.

57. Macdonald G. The Epidemiology and Control of Malaria. London UK: Oxford University Press; 1957.

58. Moreno J E, Rubio-Palis Y, Páez E, Pérez E, Sánchez V. Abundance, Biting Behaviour and Parous Rate of Anopheline Mosquito Species in Relation to Malaria Incidence in Goldmining Areas of Southern Venezuela. Medical and Veterinary Entomology. 2007;21:339-349.
59. Jones A, Uddenfeldt U, Morse A P, Hastings I M, Gagnon A S. Climate Prediction of El Niño Malaria Epidemics in North-west Tanzania. Malaria Journal. 2007;6:162.

60. Newton I. Principios Matemáticos de la Filosofía Natural. Ba rcelona: Altaya S.A.; 1993.

61. Yilmaz H. Mathematical Aids for General Relativity. En: Introduction to the Theory of Relativity and the Principles of Modern Physiscs. New York: Blaisdell Publishing Company; 1965. p. 138-145.

62. May R M. Simple Mathematical Models with Very Complicated Dynamics. Nature. 1976;261:459-67.

63. Procaccia I, Zeitak R. Shape of Fractal Growth Patterns: Exactly Solvable Models and Stability Considerations. Phys Rev Lett. 1988;60:2511.

64. Bortman M. Elaboración de Corredores o Canales Endémicos Mediante Planillas de Cálculo. Rev Panam Salud Publica. $1999 ; 5(1): 1-8$

65. Calude C S, Stay M A. From Heisenberg to Godel Via Chaitin. International Journal of Theoretical Physics. 2007;46(8):2013-25. 